
\title{
$\begin{array}{ll}\text { Research Square } & \text { They should not be considered conclusive, used to inform clinical practice, } \\ \text { or referenced by the media as validated information. }\end{array}$
}

\section{Factors influencing malaria infection in Rwanda 2010: a cross-sectional survey study using generalized structural equation modeling}

Muhammad Abu Bakar ( $\sim$ mr.abminhas@gmail.com )

Shaukat Khanum Memorial Cancer Hospital and Research Centre https://orcid.org/0000-0001-9387-6287

Rahma Fiaz

Evercare hospital Lahore

Eustasius Musenge

University of the Witwatersrand

\section{Research article}

Keywords: Generalised structural equation modelling (G-SEM), Malaria morbidity, Malaria Indicator Survey (MIS), Demographic and Health Survey (DHS)

Posted Date: November 4th, 2020

DOI: https://doi.org/10.21203/rs.2.18420/v2

License: (c) (i) This work is licensed under a Creative Commons Attribution 4.0 International License. Read Full License 


\section{Abstract}

Background Malaria is one of the primary public health concerns in the world and an important cause of morbidity and mortality in sub-Saharan Africa. Malaria morbidity is associated with poverty and vulnerability as it is not easy for the poor people to access preventive treatment and protective measures. In Rwanda, malaria prevention has become a major problem against the double-barreled burden of an overstretched health system and strained financial resources.

Methods This work was a cross-sectional survey study design based on data from Rwanda collected in 2010 through the Malaria Indicator Survey as part of the Demographic and Health Survey. The primary outcome variable was an ordinal variable with three categories; no malaria, probable malaria, and confirmed malaria cases. The outcome variable was formulated by combining rapid malaria test and confirmatory blood smear laboratory test. Statistical analysis was done using survey ordinal logistic regression modelling adjusting for random effects for direct effects and generalised structural equation modelling (G-SEM) to obtain total (direct and indirect) effects of malaria morbidity.

Results The 11,865 participants had a mean age of 22 years, and two-thirds of the participants were females (67\%). Household related variables (socioeconomic status, health insurance, age in years) showed a significant total effect on malaria infection. Socioeconomic status had the greatest total effect which was a sum of the direct and indirect effects influenced indirectly by education, health insurance and the number of rooms for sleeping in isolation.

Conclusion Poverty is still the core issue to the morbidity patterns driving the malaria epidemic in Rwanda. Access to health facility and health insurance has a high positive impact on decreasing disease as such a special focus on some regions can be an effective intervention strategy. A better understanding of the drivers of morbidity directly and/or indirectly can better target interventions to be more efficient in those affected areas.

\section{Background}

Malaria is a major public health issue in the world numbers? [1]. Globally, it was estimated that there were 660,000 deaths due to malaria of which a large proportion over $86 \%$ were in children (less than five years age) and older people (above sixty) [2]. It is a preventable and treatable infectious disease transmitted by mosquitoes, yet it kills more than one million people every year in sub-Saharan Africa. In sub-Saharan Africa, malaria is the leading cause of death in children less than five years of age and accounts for about $91 \%$ of deaths occurring in children (less than five years of age) and older people (over sixty) [1]. (, \#5; \#2) The African Leaders Malaria Alliance (ALMA), in collaboration with the nine countries (Angola, Cameroon, Chad, Congo, Gabon, Equatorial Guinea, Central African Republic, DR Congo and Sao Tomé-et-Principe) developed an African Roadmap to eliminate malaria by 2030 [1]. According to the World Health Organization (WHO) country-specific statistics, 2.2 million inhabitants out of 11.5 million people are at risk of malaria in Rwanda [3,4]

Malaria is mesoendemic (area in which a disease incidence is sufficiently high) in the low-lands and hypoendemic (area in which a disease incidence is sufficiently low) in highlands of Rwanda [5]. Globally, in endemic areas where transmission occurred in long regular seasons, infection rates were highest among children less than five years of age who had not yet established immunity to the disease, contrary to epidemic areas where malaria transmission took place in short seasons; malaria infections in all age categories was high [6]. Furthermore, a study conducted in Ruhuha region of Rwanda concluded that there was also a high risk of malaria in older age groups during long regular malaria season [7]. The reason may be that older people did not sleep under treated bed nets as compared to young ones. Another reason might be because older people stayed out longer than younger ones which made them more likely to be bitten by mosquitoes $[8,9]$.

Historically, it is believed that the most deadly malaria specie Plasmodium falciparum is prevalent in sub-Saharan Africa [10]. Consequently, malaria is hard to control in Africa due to the effectiveness of vector species and the predominance of most severe species Plasmodium falciparum [11]. Risk of malaria infection is high in rural areas of developing countries can be attributed to poverty and poor lifestyle [12]. Factors which play the major role in disease risk include proximity to the vector breeding sites, age, socioeconomic status, altitude, moderate use of control measures, low income, limited access to health facility, no vaccination program for newborn, illiteracy, land use near pools, and open houses [13-16].

A recent global report shows that due to good political commitment and better utilization of funding, a $54 \%$ of the above reduction has been experienced in African (WHO regions) [17]. As is the case in other sub-Saharan countries, in Rwanda the use of Long-Lasting Insecticidal Nets (LLIN), Indoor Residual Spraying (IRS) and treating malaria cases with Artemesinin-based combination therapy (ACT) had reduced malaria infection up to 50\% [18,19]. Therefore, it has a significant influence on the health and socioeconomic well-being of people. Therefore, this work focuses to determine the direct and indirect determinants of malaria morbidity in poorer/financially challenged households and at an individual level in Rwanda in 2010 using malaria indicator survey (MIS) data that was accessible from the Demographic and Health Survey (DHS) website.

\section{Methodology}

This study was conducted as a cross-sectional survey design. The dataset used in this work is known as the Malaria Indicator Survey (MIS) dataset of Rwanda which was part of the Demographic and Health Surveys (DHS) 2010. This study includes both males and females tested for malaria diagnosis (rapid malaria test and confirmatory blood smear test) either positive or negative result. This work was conducted in Rwanda, a Central African country located South of the equator between latitude $1^{\circ} 4^{\prime}$ and $2^{\circ} 51^{\prime}$ South and longitude $28^{\circ} 63^{\prime}$ and $30^{\circ} 54^{\prime}$ East. It has a surface area of 26,338 square kilometers and is bordered by Uganda to the North, Tanzania to the East, the Democratic Republic of the Congo to the West and Burundi to the South. Landlocked, Rwanda lies 1,200 kilometers from the Indian Ocean and 2,000 kilometers from the Atlantic Ocean [20]. Rwanda is divided into five geographically-based provinces-North, South, East, West and the City of Kigali, with the provinces, further subdivided into 30 districts, 416 sectors, 2,148 cells, and 14,837 villages [20]. 
This study was granted ethical approval by the University of the Witwatersrand's Human Research Ethics Committee (Medical) (Clearance Certificate No. M151040). Approval to use the MIS data was obtained from the Measure DHS website. The primary study, where the data was collected, verbal informed consent for testing of children was obtained from the child's parent or guardian at the end of the household interview and ethical clearances with the Rwanda authorities before the study started.

The data used for the analysis was obtained from the 2010 Malaria Indicator Survey (MIS) conducted by Rwanda Demographic and Health Survey (RDHS) program. The previous study collected the data elements on basic demographic and health indicators, malaria prevention, treatment and morbidity.

\section{Dependent variable}

The malaria outcome variable for this study was defined according to the Center for Disease Control (CDC) and World Health Organization case definition criteria. The CDC and WHO definition indicates that there are three possible states of malaria infection $[21,22]$.

\section{No malaria}

2. Probable or (symptomatic or asymptomatic) malaria infection

3. Confirmed malaria infection

Two types of tests were conducted on surveyed population (rapid malaria test and confirmatory blood smear laboratory test). Participants who showed a negative result in both the tests (rapid malaria test and confirmatory blood smear laboratory test) were put in the category of "No Malaria Cases". The participants who were not tested for confirmatory blood smear laboratory or either show negative test but showed positive in the rapid malaria test were considered as "Probable or symptomatic or asymptomatic Malaria Cases". Those who have positive confirmatory blood smear laboratory test regardless of their results from rapid malaria test either positive or negative were considered as confirmed malaria cases.

\section{Independent variables}

The independent variables were split into four categories: individual level variables (education gain in years, age in years, social economic status, health insurance, household related variable (no of rooms for sleeping), ecological variables (cluster altitude in meters and region) and behavioural variables (has clean water facility for drinking, and sleep under bed nets).

Factor analysis (FA) was used to derive socio-economic status (SES) using indicators data from 2010 Rwanda Demographic Health Survey (RDHS). Factor analysis is a useful tool for investigating variables relationships for complex concepts such as socioeconomic status[23]. It allows researchers to investigate concepts that are not easily measured directly by collapsing a large number of variables into a few interpretable underlying factors. The process to create socio-economic status is shown in (Appendix S1 and S2).

\section{Conceptual framework}

Reiter P. in 2008 studied the effects of temperature on Malaria transmission. His study proposed that temperature, rainfall and humidity cannot be considered in exclusion without considering the behavior of human. Additional factors that influence the malaria infection directly and/or indirectly; household and individual level related variables (SES, age, education and health insurance), behavioural variables (Clean water facility for drinking and sleep under bednet), ecological variables [Regions (north, east, west and south)] and cluster altitude in meters).

This study is considering all the direct and indirect factors with the inclusion of human behavior as suggested by Reiter P. in 2008 [24]. This work not only considers human behavior but related direct and indirect factors (mentioned above) which have not been considered in previous studies.

Conceptual modelling technique and variables are divided into three main categories; ecological, household and/or individual level, and behavioural variables in the analysis with the purpose that these determinative factors run through the standard set of either 'proximate direct or indirect variables that have an influence on malaria morbidity $[25,26]$. Variables are either endogenous (dependent variable) or exogenous (explanatory variable) or both, which can be modelled by using generalised structural equation models. Details of these models provided in Figure 4 and Figure 5.

\section{Statistical analysis}

Power computation was done using a STATA ado-file. [27] A sample prevalence of $2.1 \%$ comprised of $1.4 \%$ children and $0.7 \%$ adults adopted from the Rwanda Malaria Indicator Survey [3] and a 3\% assumed population prevalence at an alpha level of 0.05. A design effect of 10 was used and intercluster correlation (ICC) of 0.071 with a total of 492 clusters and average households per cluster of 115 . Power was obtained an $89 \%$. Descriptive analysis was conducted using survey chi square test (Rao-Scott adjustment) adjusted for cluster effect. For of categorical variables weighted percentages (proportion) with adjusted F-statistic are reported as shown in Table 1. For continuous variables mean and confidence interval are reported as shown in Table 1. Bivariate analysis was done using survey chi-square test adjusting for cluster effect to establish the relationship between two categorical variables (such as malaria morbidity and gender). For the continuous explanatory variables such as age, survey ordinal univariate analysis adjusted for cluster effect was done. For bivariate analysis odds ration with p-value are reported in Table 2.

Multivariate analysis was done using step-wise forward selection survey ordinal logistic regression modelling adjusting for random effects and generalised structural equation modelling (G-SEM) to obtain total (direct and indirect) effects of malaria infection. The models were also tested using the ordinal regression diagnostics i.e. goodness of fit and multicollinearity; as those variables which were correlated with each other deleted from the final model. Variables were deleted from the model if they showed multicollinearity. Suest test was used to check the goodness of fit and see if the addition of a variable did improve the model. It checks the estimates and (co)variance of the coefficients of two models simultaneously and gives adjusted p-value. The selection 
was based on variables that were most significant in the final survey ordinal regression model. The model fit was assessed using the root mean square error of approximation (RMSEA) due to its sensitivity to the number of estimated model parameters and ability to handle large samples [29]. Studies show that an RMSEA below 0.8 shows evidence of good fit, [29] hence the RMSEA of 0.03 from our G-SEM model was a good fit. All statistical analyses were carried out using Stata ${ }^{\circledR} 13.1$ (Copyright 1985-2013, StataCorp LP) Arc GIS was used for mapping the prevalence and variance of malaria infection in Rwanda.

\section{Results}

\section{Descriptive statistics}

The total number of individuals participated in this study was 11 , with a mean age and standard deviation of $22 \pm 18$ years. The number of individuals who were tested for malaria using malaria blood smear test and rapid malaria test, of whom 11,610 (97.67\%) had no malaria cases, 137 (1.26\%) had probable malaria cases, and $118(1.07 \%)$ had definite malaria cases. The Eastern region had weighted percentage of (0.64) of the total definite cases, the Southern region had $(0.26)$ of the total definite cases, the Western region had $(0.0008)$ of total definite cases, and the Northern and the Kigali region had like ( 0.0007$)$ of total definite cases. A total of weighted percentage of (1) of individuals having definite malaria cases were from rural areas and (0.0005) was from urban areas. Female individuals were approximately (0.69), and male individuals were (0.36) had definite weighted percentages of malaria cases of the total population. Furthermore, Figure 2-3 showed the mean prevalence and variance of malaria infection in Rwanda 2010 using the direct and indirect approach. Musanze, Nyabihu and Ngororero regions had the greater prevalence of malaria infection in Rwanda in 2010 (Figure 2). Nyagatara region had the greater variance of malaria infection in Rwanda in 2010 (Figure 3).

The weighted mean and confidence interval of education gain in years and cluster altitude were $1.38(0.89,1.86)$ and $1524(1483.57,1565.61)$ in meters. The descriptive statistics survey weighted percentages of independent variables with the significant test statistic of survey adjusted chi-square test are summarized in Table 1. The results of survey ordinal univariate analysis, survey ordinal logistic regression modelling adjusting for random effects for random effects for direct effects and generalised structural equation modelling (G-SEM) to obtain (direct and indirect) effects of malaria infection are summarized in Table 2.

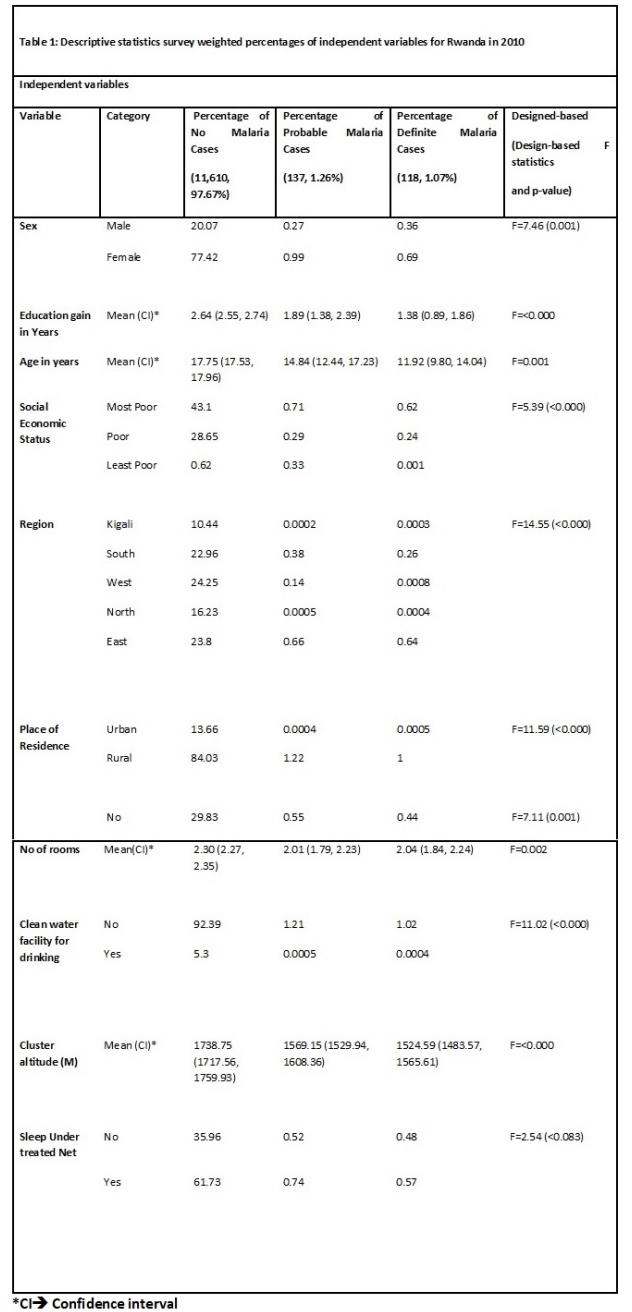




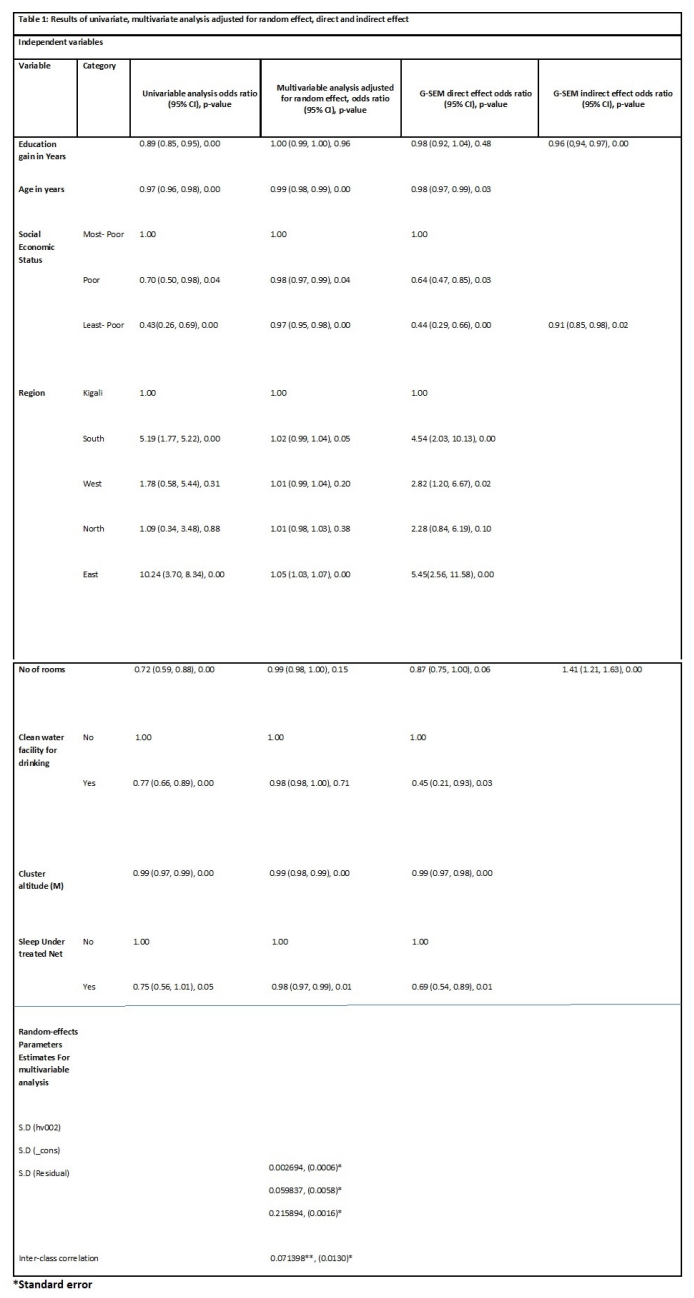

** Interclass correlation $=\frac{s d(h v 002)^{2}+s d(\text { cons })^{2}}{s d(h v 002)^{2}+s d(\text { cons })^{2}+s d(\text { residual })^{2}}=\frac{\left(0.002694^{2}+(0.0 .05983)^{2}\right.}{\left(0.002694^{2}+(0.0 .05983)^{2}+\left(0.215894^{2}\right.\right.}=\mathbf{0 . 0 7 1 3 9 8}$

\section{Result of direct, indirect and total effects using (G-SEM)}

\section{Results of direct effects}

The direct effects from G-SEM are shown in Table 3. The arrows show pathways that were statistically directly significant. Household related variables (number of rooms for sleeping, socio-economic status, health insurance, age in years and education gain in years), has significantly shown direct effect on malaria infection. Ecological variables (region and cluster altitude in meters) and behavioural variables (sleep under treated bed net and clean water facility for drinking) also showed significant direct effect on malaria infection.

Household related variables, least poor (a category of SES) and health insurance were modeled as an exogenous variable, which directly affected negatively on malaria infection as shown in Table 3 adjusting for other household level variables. The number of rooms for sleeping was modeled as an exogenous variable, directly impacted negatively on malaria infection as shown in Table 3 with total effect as shown in Table 3.

\section{Results of indirect effects}

The indirect effects found from G-SEM are shown in Table 3. The connected arrows show indirect pathways that were statistically significant. Moreover, variable like the number of rooms for sleeping was indirectly influenced by least poor and least poor was indirectly affected by education gain in years. Education gain in years also affected the health insurance which showed the positive relationship as shown in Table 3 . Furthermore, when health insurance was treated as an endogenous variable, SES variable category "least poor" indirectly impacted malaria infection and had a protective effect as shown in Table 3. Education in years as an exogenous variable had no direct influence on malaria infection, but indirectly affected malaria infection through least poor as shown in Table 3 and had a protective effect on health insurance as shown in Table 3. When the number of rooms for sleeping was treated as an endogenous variable, SES variable category least poor was indirectly negatively impacted the malaria infection. However, it positively effected when the number of rooms for sleeping effect was direct as shown in Table 3. 


\section{Total effect}

The total effect is an accumulative value of direct and indirect effects of each respective variable. The results of the total effect are shown in Table 3. Least poor category showed a significant total effect of as shown in Table 3 Health insurance also showed a total effect of as shown in Table 3 . Education gain in years showed an indirect effect and resulting total effect was as shown in Table 3.

\begin{tabular}{|c|c|c|c|c|c|c|}
\hline \multirow{2}{*}{ Variable Category } & \multicolumn{4}{|c|}{ Direct Effect on Malaria infection in children Rwanda 2010} & \multirow{2}{*}{$\begin{array}{l}\text { Indirect Effect on child } \\
\text { Malaria infection }\end{array}$} & \multirow{2}{*}{$\begin{array}{l}\text { Total Effect on child Malaria } \\
\text { infection }\end{array}$} \\
\hline & Education & Least Poor & \begin{tabular}{|l} 
No. of \\
Rooms
\end{tabular} & $\begin{array}{l}\text { Malaria Diagnosis } \\
\text { direct effect coef. }\end{array}$ & & \\
\hline Education in Years & & & & & $-0.04074(0.00836)^{*}$ & $-0.04074^{* *}(0.00836)^{*}$ \\
\hline Age in years & & & & $-0.01869(0.00479)^{*}$ & & $-0.01869 * *(0.00479)^{\bullet}$ \\
\hline \multicolumn{7}{|l|}{ Social Economic Status } \\
\hline Poor & & & & $-0.46468(0.15131)^{\bullet}$ & & \\
\hline Least Poor & $\begin{array}{l}0.04114 \\
(0.00056)^{*}\end{array}$ & & $\begin{array}{l}0.48576 \\
(0.00907)^{*}\end{array}$ & $-0.84267(0.20614)^{*}$ & $-0.08464(0.03717)^{*}$ & $-0.92720^{* *}(0.20212)^{*}$ \\
\hline \multicolumn{7}{|l|}{ Region } \\
\hline South & & & & $1.52598(0.40979)^{*}$ & & $1.52598^{* *}(0.40979)^{*}$ \\
\hline West & & & & $1.05460(0.43893)^{\bullet}$ & & $1.05460^{* *}(0.43893)^{*}$ \\
\hline North & & & & $0.84064(0.50849)^{*}$ & & $0.84064^{* *}(0.50849)^{*}$ \\
\hline East & & & & $1.71304(0.38413)^{\bullet}$ & & $1.71304^{* *}(0.38413)^{*}$ \\
\hline Cluster altitude in meters & & & & $-0.00337(0.00044)^{*}$ & & $-0.00337^{* \bullet}(0.00044)^{*}$ \\
\hline Sleep Under treated Net & & & & $-0.36210(0.13021)^{*}$ & & $-0.36210^{* *}(0.13021)^{*}$ \\
\hline Health Insurance & $\begin{array}{l}0.01109 \\
(0.00063)^{*}\end{array}$ & $\begin{array}{l}0.06660 \\
(0.00472)^{*}\end{array}$ & & $-0.23371(0.13173)^{*}$ & & $-0.3580 \cdots 0.1396)^{*}$ \\
\hline $\begin{array}{l}\text { Number of rooms for } \\
\text { sleeping }\end{array}$ & & & & $-0.14219(0.07511)^{*}$ & & $-0.23297^{* *}(0.131793)^{*}$ \\
\hline $\begin{array}{l}\text { Clean water facility for } \\
\text { drinking }\end{array}$ & & & & $-0.80843(0.20614)^{*}$ & & $-0.34357^{* *}(0.07566)^{*}$ \\
\hline
\end{tabular}

\section{*Standard error}

\section{** Total effect}

** Total effects computed as the product along the related pathways of least poor, i.e. $((0.06660) *(-0.23371))+((0.48576) *(-$ $0.23371))+(--0.84267)=-0.92720(0.20212)$

\section{Results of direct and indirect generalised structural equation modelling (G-SEM)}

Generalised structural equation modelling (G-SEM) was based on variables which were statistically significant in regression analyses were chosen for G-SEM pathways as shown in Figure 4-5. Results were reported adjusting endogenous and exogenous factors and keeping other factors constant. The results of direct and indirect G-SEM technique showed positive and/or negative effects on the endogenous malaria infection.

Exogenous variables were least poor, health insurance, regions (north, east, west and south), and a number of rooms for sleeping in a household. The indirect effects were modelled on variables education in years, health insurance, least poor and a number of rooms for sleeping.

Figure 4 highlights the direct pathways of malaria infection (arrows directly linked to the brown square) and the indirect pathways were showed all possible routes of malaria in Figure 5.

Education gain in years indirectly impacted the malaria infection in two ways least poor (protective) and health insurance (a risk) as shown in Figure 5 .

$$
\begin{aligned}
& \text { Education }=>\text { least poor }=>\text { malaria infection }=\text { Protective } \\
& \text { Education }=>\text { health insurance }=>\text { malaria infection }=\text { Risk }
\end{aligned}
$$

Eastern region showed greatest total effect on malaria infection as shown in Table 3, and cluster altitude in meters showed least total effect on malaria infection as shown in "Table 3.

The result of G-SEM showed both direct and indirect effects on endogenous variable malaria infection. The direct G-SEM model (Figure 4) and indirect G-SEM model is (Figure 5) are shown. The endogenous variables; least poor, the number of rooms for sleeping, and health insurance are shown in Figure 5.

\section{Discussion}

The current study revealed that malaria infection was influenced by the combination of variables such as behavior, household condition and ecological factors. In 2015, 214 million deaths were recorded due to malaria and 91 \% were in sub-Saharan Africa [30]. Social-economic status was a fundamental factor of malaria morbidity in several studies [31,32]. The studies also provided a positive malaria diagnosis. 
As mentioned earlier, malaria is associated with poverty [31]. The findings of this work complement the results of the previous studies on malaria morbidity. Previous studies have shown that low socio-economic status increases the prevalence of malaria infection in developing countries [33]. According to the United Nations Accounts (UNA) central aggregated database 2015, Rwanda has low Gross Domestic Product (GDP) [34] which translates into higher malaria infections. This study also confirms the finding. It has also been indicated that the malaria infection is a disease of developing countries putting an additional burden on the healthcare facilities.

Logically the nutritional level is linked with GDP. Therefore, it is obvious that the poorer countries have inadequate resources to improve nutritional levels resulting in poor immunity against malaria disease [15]. Poor SES results into an insufficient use of health-care facilities, therefore increasing the vulnerability of the population to the risk of malaria. Governments of the poorer countries need rational, optimum and affordable policies to control malaria infection and treatment. This could be achieved by investing in research and data collection to investigate the trends of malaria to make effective and efficient policies and proper utilisation of resources.

Regions (Kigali, south, north, east and west) place-of-residence (urban or rural) and cluster altitude in meters may be interrelated. However, fewer studies indicated an effect on malaria prevalence dependence on altitude [35-37]. Researchers also revealed that individuals living in low altitude areas are more at risk as compared to higher altitude areas [38]. In this work, altitude has shown a marginal reduction in malaria infection. A study conducted in Thailand concluded that school children could be a better source of anti-malaria education for the family members in contrast to disseminating messages by newsletters [39].

To achieve significant reduction in malaria morbidity in Rwanda, there is a need of improvement in the awareness and education status of the targeted population [40]. Knowledge about malaria prevention might be conveyed in the community by the students, who facilitate the household and families by applying and following influence in the targeted settings. In this study, Age has shown a significance ( $p$-value $=0.001)$. Previous studies have shown that children over the age of five were less at risk of malaria infection $[8,41]$. In this work, increase in age has shown a reduced tendency of malaria infection hence it is less likely to have positive malaria diagnosis as the age increases. Existing literature also confirms that older people (above 50) have lesser chance of malaria infection due to developed immunity.

Use of treated bed net have shown significant effect ( $p$-value $=0.004)$. Results of this study show that individuals who sleep under a treated bed net, are less likely to have positive malaria diagnosis test. It might be due to the fact that they have a lesser chance of having direct contact with infected mosquitos.

Health insurance showed marginal significance of $p$-value $=0.06$. The results of the study show that people with health insurance are less likely to get the malaria infection. It is possibly due to health related companies or knowledge of disease provided by the health insurance companies. Health insurance companies also provide preventative methods to protect against malaria.

Participants who have clean water facility for drinking, are less likely of contracting malaria as shown with significant $p$-value $=0.01$. The possibilities are that the malaria is an infectious disease and contaminated water is a good inhabitant for mosquito. Therefore, participants who have clean water supply for drinking may be protected/safe as compared who do not.

The number of rooms for sleeping have shown significance $(p$-value $=0.01)$. Hence, people who have more rooms for sleeping they are less likely of getting malaria infection.

The G-SEM's indirect pathways showed a significant association between SES and health insurance, education and health insurance, education and least poor as well as between SES and number of room for sleeping level. G-SEM was used in this study to complement the results from the multiple variable analyses. The results showed that the multiple variable analysis and the G-SEM direct pathways show similar results.

G-SEM can help in diagrammatically measuring the effects of the determinants of the outcome and this can assist in the analysis where the variables can be separated into those with a direct effect on the outcome and those with an indirect effect on the outcome. This will help to explain better some factors that might not directly affect the outcome, and inform policy on adopting indirect and direct approaches to dealing with the malaria infection in children and adults.

\section{Conclusions}

This study shows the importance of socio-economic status as well as influence of education in the fight against malaria. To eliminate malaria morbidity in the population, it is important for the governments to empower the community economically, intellectually and ensure the health education and awareness is a part of the efforts to fight the endemic. Access to health insurance has a positive impact on decreasing malaria infections. Therefore health insurance could be therefore focused as an effective tool in the intervention strategy especially in relatively high income sectors to significantly reduce the infections. This will assist in the fight to eliminate malaria.

It is important to ensure that the resources are channeled to optimize prevention strategies that are put in place. Once the population is empowered, the preventative strategies can then be implemented successfully. If the population is educated, it can understand the strategies put in place and follow them successfully. Health authorities and Government have to have latest knowledge of the determinant factors in order to plan preventive and curative strategies of malaria. The methods of analysis used in this work could help the health authorities to make effective and efficient strategies against malaria. Hence public health awareness campaigns must be empowered to educate the masses and eliminate malaria morbidity. It is necessary to understand the direct and indirect factors of malaria morbidity so that effective monitoring and evaluation policies for malaria control can be formulated. 
The reduction in poverty will go a long way in the fight to eradicate malaria in Africa in particular and globally in general. Generalized structural equation modeling (G-SEM), Malaria morbidity, Malaria indicator survey (MIS), Demographic and health survey (DHS), Socio-economic status (SES), Infectious disease, African Leaders Malaria Alliance (ALMA), Long-lasting insecticidal nets (LLIN), Artemisia combination therapy (ACT), National Indicator Survey of Rwanda (NISR), World Health Organization (WHO), Factor Analysis (FA), Gross Domestic Product (GDP), United Nation (UN).

\section{Abbreviations}

Generalized structural equation modeling (G-SEM), Malaria morbidity, Malaria indicator survey (MIS), Demographic and health survey (DHS), Socio-economic status (SES), Infectious disease, African Leaders Malaria Alliance (ALMA), Long-lasting insecticidal nets (LLIN), Artemisia combination therapy (ACT), National Indicator Survey of Rwanda (NISR), World Health Organization (WHO), Factor Analysis (FA), Gross Domestic Product (GDP), United Nation (UN).

\section{Declarations}

\section{ACKNOWLEDGEMENT}

This work was performed as part of the Master's degree in the field of Epidemiology and Biostatistics in the School of Public Health, Faculty of Health Sciences at the University of the Witwatersrand Johannesburg, South Africa.

\section{References}

1. Roll back malaria. Available at: http://www.rollbackmalaria.org/about/about-malaria/what-is-malaria 2 October 2016].

2. Kundu R, Ganguly N, Ghosh TK, Choudhury P, Shah RC (2005) Diagnosis and management of malaria in children: recommendations and IAP plan of action. Indian Pediatr 42: 1101-1114.

3. World Health Organization (Rwanda: Country Health Profile). Available from: http://www.who.int/countries/rwa/en/. Accessed 8 July 2016.

4. National Institute of Statistics of Rwanda. Fourth Population and Housing Census. Thematic Report: Characteristics of households and housing. Ministry of Finance and Economic Planning; 2012. Available from: http://statistics.gov.rw/file/2907/download?token=i09m0Bly. Accessed 7 July 2016.

5. President's Malaria Initiative. Malaria Operational Plan (MOP). Kigali, Rwanda: Rwanda FY 2014; 2014 [cited 2 May 2016 ].

6. Feachem RG, Phillips AA, Hwang J, Cotter C, Wielgosz B, et al. (2010) Shrinking the malaria map: progress and prospects. Lancet 376: $1566-1578$.

7. Smith T, Hii JL, Genton B, Muller I, Booth M, et al. (2001) Associations of peak shifts in age--prevalence for human malarias with bednet coverage. Trans R Soc Trop Med Hyg 95: 1-6.

8. Winskill P, Rowland M, Mtove G, Malima RC, Kirby MJ (2011) Malaria risk factors in north-east Tanzania. Malar J 10: 98.

9. Lengeler C (2004) Insecticide-treated bed nets and curtains for preventing malaria. Cochrane Database Syst Rev: Cd000363.

10. Cheesbrough Monica (2006). District laboratory practice in tropical countries. New York-Cambridge press Part1, 2nd edition Pp249-258. Arora DR and Arora B (2008). Medical Parasitology. 2nd edition.Pp.67-76.

11. Coluzzi M (1999) The clay feet of the malaria giant and its African roots: hypotheses and inferences about origin, spread and control of Plasmodium falciparum. Parassitologia 41: 277-283.

12. Donnelly MJ, McCall PJ, Lengeler C, Bates I, D'Alessandro U, et al. (2005) Malaria and urbanization in sub-Saharan Africa. Malar J 4: 12.

13. Stratton L, O'Neill MS, Kruk ME, Bell ML (2008) The persistent problem of malaria: Addressing the fundamental causes of a global killer. Social Science \& Medicine 67: 854-862.

14. Yamamoto S, Louis V, Sié A, Sauerborn R (2010) Household risk factors for clinical malaria in a semi-urban area of Burkina Faso: a case-control study. Transactions of the Royal Society of Tropical Medicine and Hygiene 104: 61-65.

15. Caulfield LE, Richard SA, Black RE (2004) Undernutrition as an underlying cause of malaria morbidity and mortality in children less than five years old. Am J Trop Med Hyg 71: 55-63.

16. Abeku TA, van Oortmarssen GJ, Borsboom G, de Vlas SJ, Habbema J (2003) Spatial and temporal variations of malaria epidemic risk in Ethiopia: factors involved and implications. Acta tropica 87: 331-340.

17. WHO (2014) World malaria report 2014. Available from: http://rbm.who.int/wmr2014/ [cited 2 May 2015].

18. Otten M, Aregawi M, Were W, Karema C, Medin A, et al. (2009) Initial evidence of reduction of malaria cases and deaths in Rwanda and Ethiopia due to rapid scale-up of malaria prevention and treatment. Malar J 8: 14.

19. Karema C, Aregawi MW, Rukundo A, Kabayiza A, Mulindahabi M, et al. (2012) Trends in malaria cases, hospital admissions and deaths following scale-up of anti-malarial interventions, 2000-2010, Rwanda. Malaria journal 11: 236.

20. Ministry of Health, National Malaria Control Programme, MEASURE DHS, ICF International: Rwanda Malaria Indicator Survey (MIS) 2010. https://dhsprogram.com/pubs/pdf/MIS16/MIS16.pdf. Assessed 8 July 2016.

21. Center for disease control. Available from: https://wwwn.cdc.gov/nndss/conditions/malaria/case-definition/2014/. [Cited 6 January 2016].

22. ( World Health Organization. Annex 1. Defining the term "malaria case". Available from: http://www.who.int/malaria/mpac/mpac-sept2015-terminologyannex1.pdf [Cited 10 January 2017].).

23. Gwatkin DR, Rutstein S, Johnson K, Pande R, Wagstaff A (2000) Socio-economic differences in health, nutrition, and population. Washington, DC: World Bank. 
24. Reiter P (2008) Global warming and malaria: knowing the horse before hitching the cart. Malar J 7 Suppl 1: S3.

25. Mosley WH, Chen LC (1984) An analytical framework for the study of child survival in developing countries. Population and development review 10: 2545.

26. Lewis JJ, Donnelly CA, Mare P, Mupambireyi Z, Garnett G, et al. (2007) Evaluating the proximate determinants framework for HIV infection in rural Zimbabwe. Sexually transmitted infections.

27. Stata ado-file st02862: A menu-driven facility for sample-size calculations in cluster randomized controlled trials. K. Hemming and J. Marsh. Stata Journal 14: 701; 13: 114-135.

28. Grace JB (2008) Structural equation modeling for observational studies. The Journal of Wildlife Management 72: 14-22.

29. Hooper D, Coughlan J, Mullen M (2008) Structural equation modelling: guidelines for determining model fit. Articles: 2.

30. Roll back malaria. Available from: http://www.rollbackmalaria.org/about/about-malaria/what-is-malaria [cited 2 May 2015].

31. Snow RW, Craig MH, Newton C, Steketee RW (2003) The public health burden of Plasmodium falciparum malaria in Africa. Working Paper 11. Disease Control Priorities Project, Fogarty International Center, National Institutes of Health, Bethesda

32. President's Malaria Initiative. Malaria Operational Plan (MOP). Kigali, Rwanda: Rwanda FY 2014; 2014 [cited 2 May 2015 ].

33. Sachs J, Malaney P (2002) The economic and social burden of malaria. Nature 415: 680-685.

34. Map of Rwanda. Available from: https://www.google.co.za/search? q=Rwanda+map\&rlz=1C1GNAM_enZA681ZA681\&source=Inms\&tbm=isch\&sa=X\&ved=0ahUKEwiJruC5rN30AhWD7RQKHRmSDXwQ_AUICCgB\&biw=144C . Assessed 8 August 2016.

35. Drakeley CJ, Corran PH, Coleman PG, Tongren JE, McDonald SL, et al. (2005) Estimating medium- and long-term trends in malaria transmission by using serological markers of malaria exposure. Proc Natl Acad Sci U S A 102: 5108-5113.

36. Hay SI, Guerra CA, Tatem AJ, Noor AM, Snow RW (2004) The global distribution and population at risk of malaria: past, present, and future. Lancet Infect Dis 4: 327-336.

37. Lindsay SW, Martens WJ (1998) Malaria in the African highlands: past, present and future. Bull World Health Organ 76: 33-45.

38. Abeku TA, van Oortmarssen GJ, Borsboom G, de Vlas SJ, Habbema JD (2003) Spatial and temporal variations of malaria epidemic risk in Ethiopia: factors involved and implications. Acta Trop 87: 331-340.

39. Okabayashi H, Thongthien P, Singhasvanon P, Waikagul J, Looareesuwan S, et al. (2006) Keys to success for a school-based malaria control program in primary schools in Thailand. Parasitol Int 55: 121-126.

40. O'Meara WP, Mangeni JN, Steketee R, Greenwood B (2010) Changes in the burden of malaria in sub-Saharan Africa. Lancet Infect Dis 10: $545-555$.

41. Mawili-Mboumba DP, Bouyou Akotet MK, Kendjo E, Nzamba J, Medang MO, et al. (2013) Increase in malaria prevalence and age of at risk population in different areas of Gabon. Malar J 12: 3.

\section{Figures}




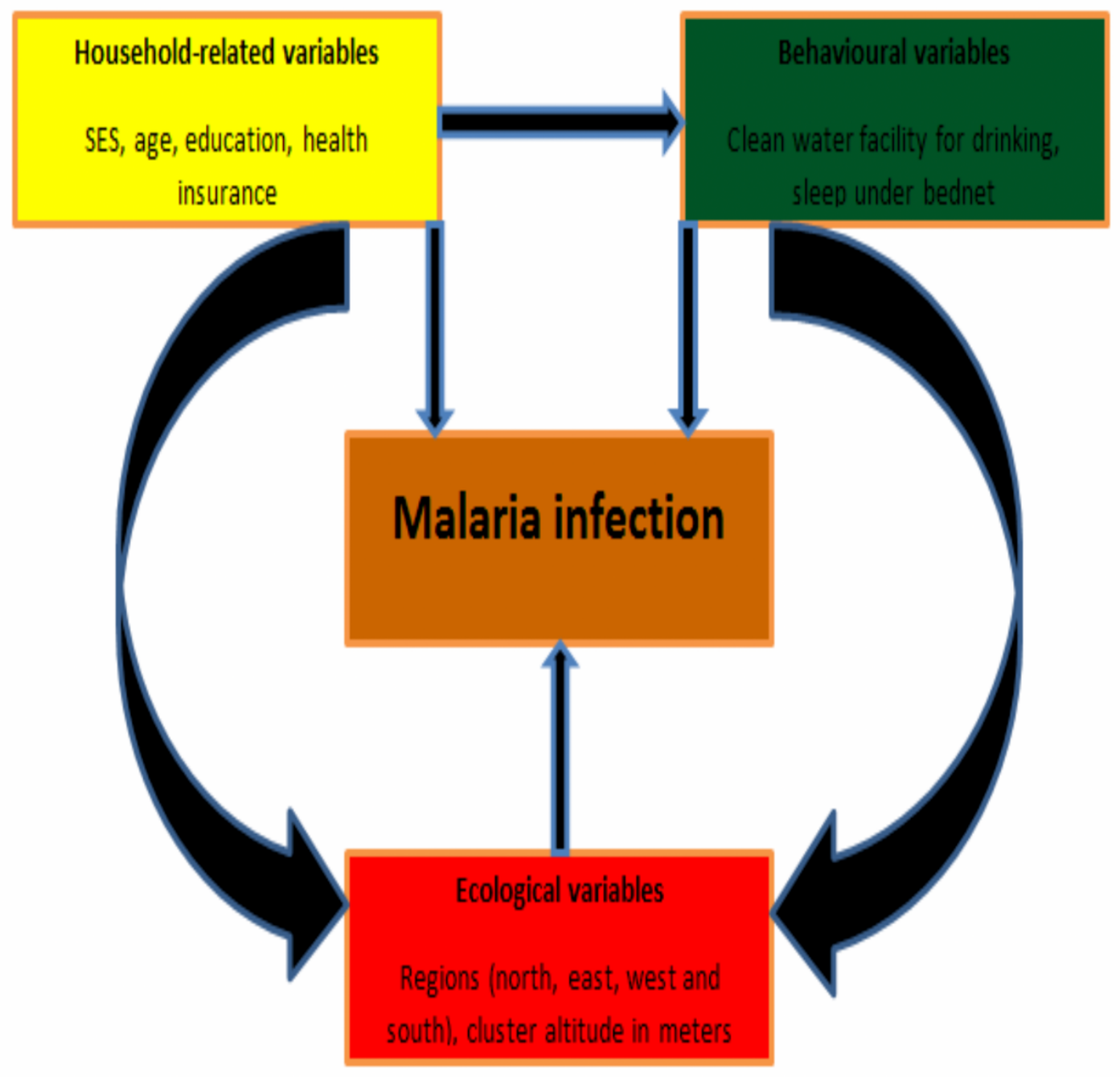

Figure 1

Conceptual frameworks for modelling malaria morbidity. The black arrows reflect a direct and indirect effect on malaria morbidity 


\section{A}

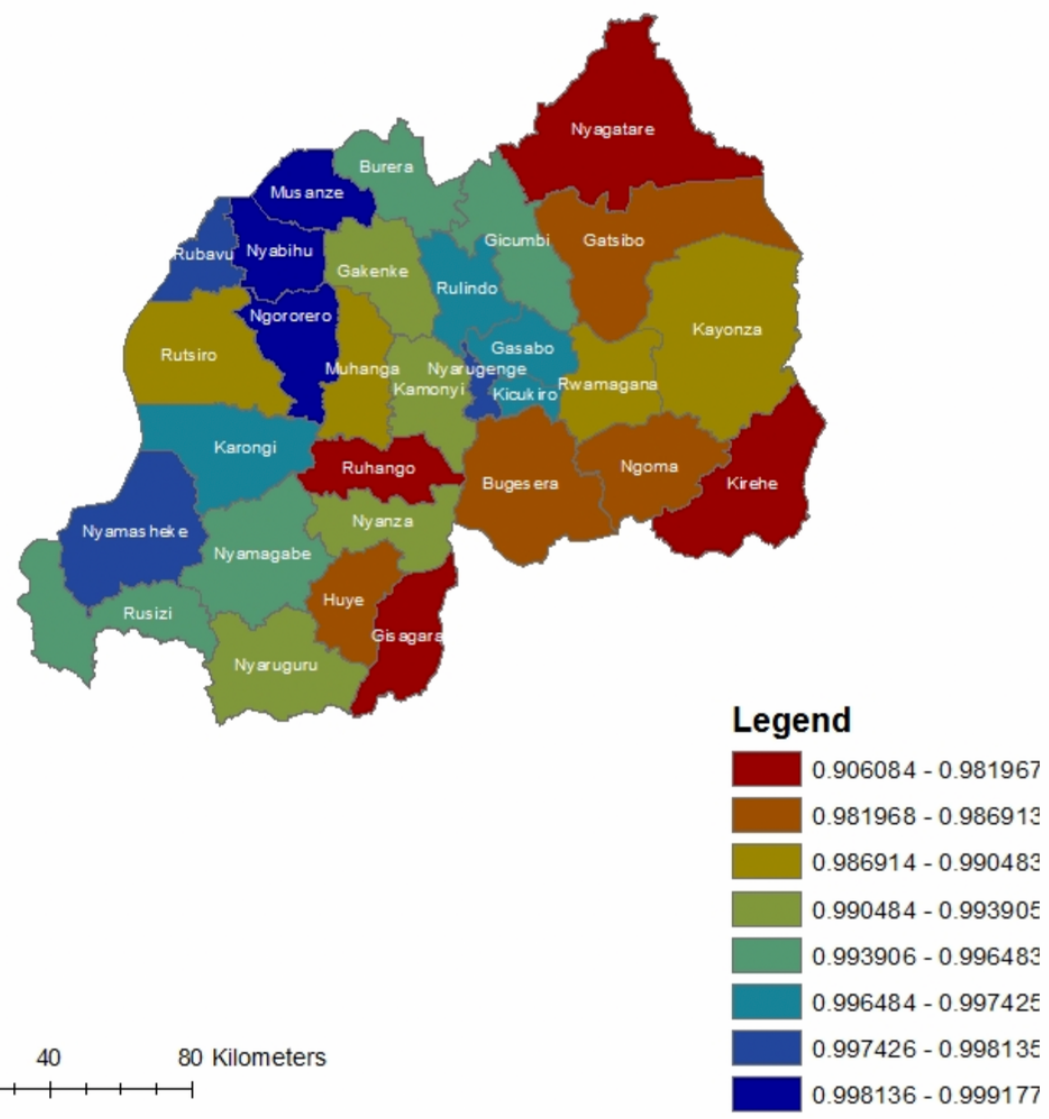

Figure 2

Mean prevalence of malaria infection using direct and indirect G-SEM approach in Rwanda in 2010 (ArcGIS 10.1. was used for mapping the prevalence in Rwanda). 


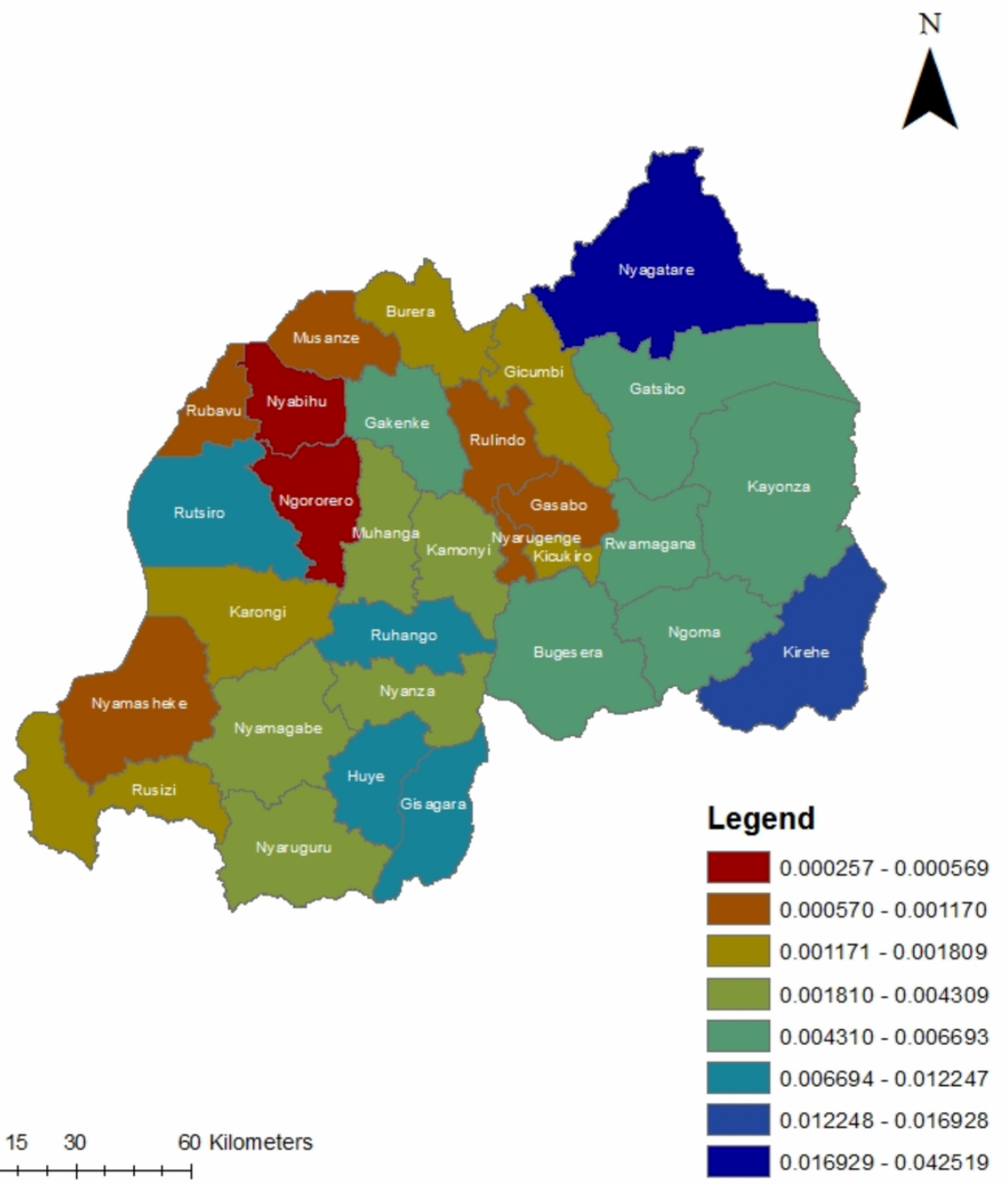

\section{Figure 3}

Variance of malaria infection using direct and indirect G-SEM approach in Rwanda in 2010 (ArcGIS 10.1. was used for mapping the prevalence in Rwanda). 


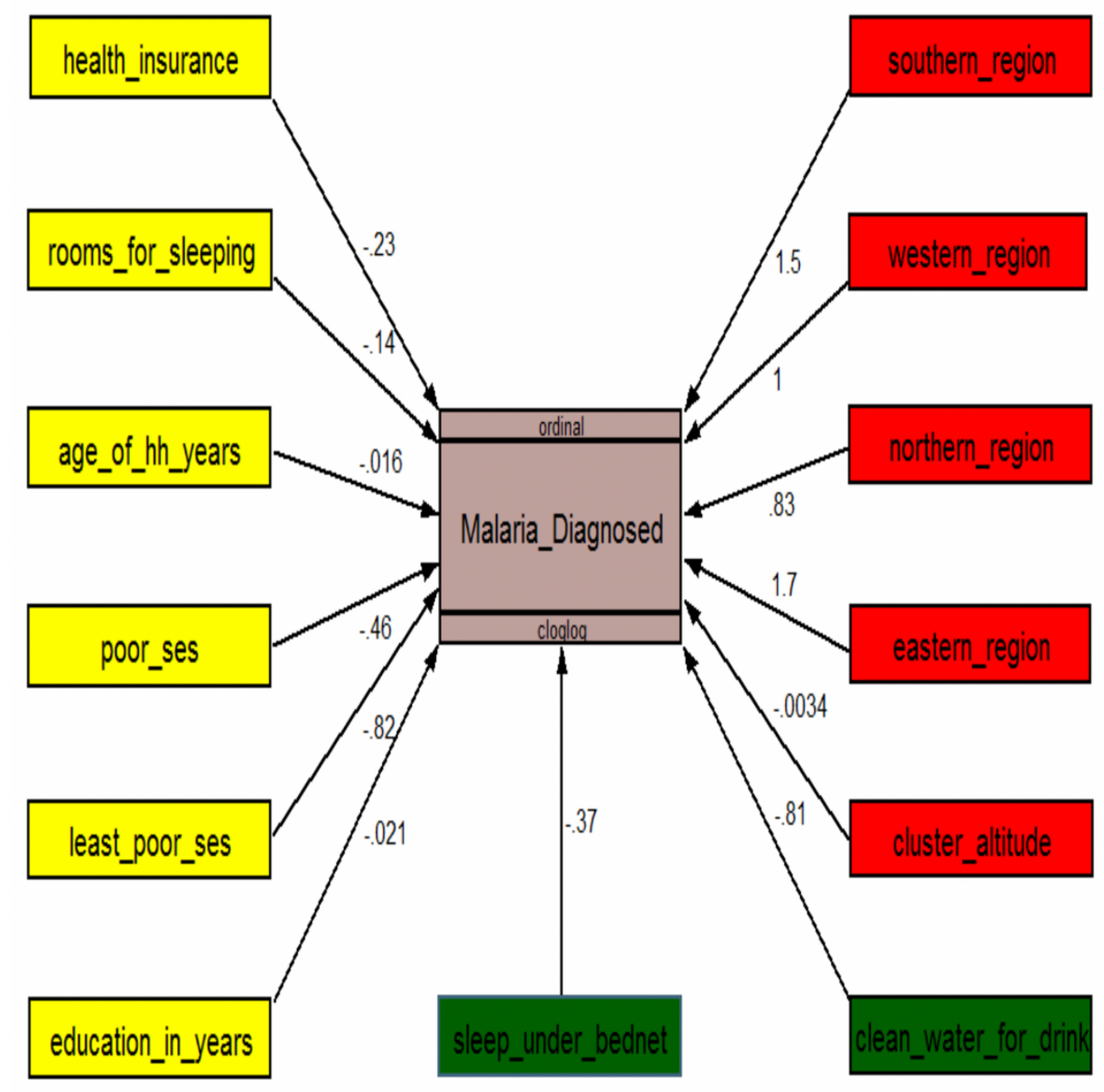

\section{Figure 4}

G-SEM pathway diagram shows direct coefficients from ordinal regression analysis of the effects of selected random predictor variables on malaria infection outcome variable result in children and adults in Rwanda in 2010. Key for variables names: individuals covered by health insurance (health_insurance), number of rooms for sleeping in each house (rooms_for_sleeping), age of household members in years (age_of_hh_years), poor SES versus most poor SES (poor_ses), least poor SES versus most poor SES (least_poor_ses), education gain of each household member in years (education_in_years), number of household members sleep under treated mosquito bed net (sleep_under_bednet), household members have a clean water excess for drinking (had_water_excess), cluster altitude in meters (cluster altitude), eastern region of Rwanda (eastern_region), northern region of Rwanda (northern_region), western region of Rwanda (western_region), southern region of Rwanda (southern_region). Other: the arrows pointing from the exogenous (explanatory variables) to endogenous (dependent variable). 


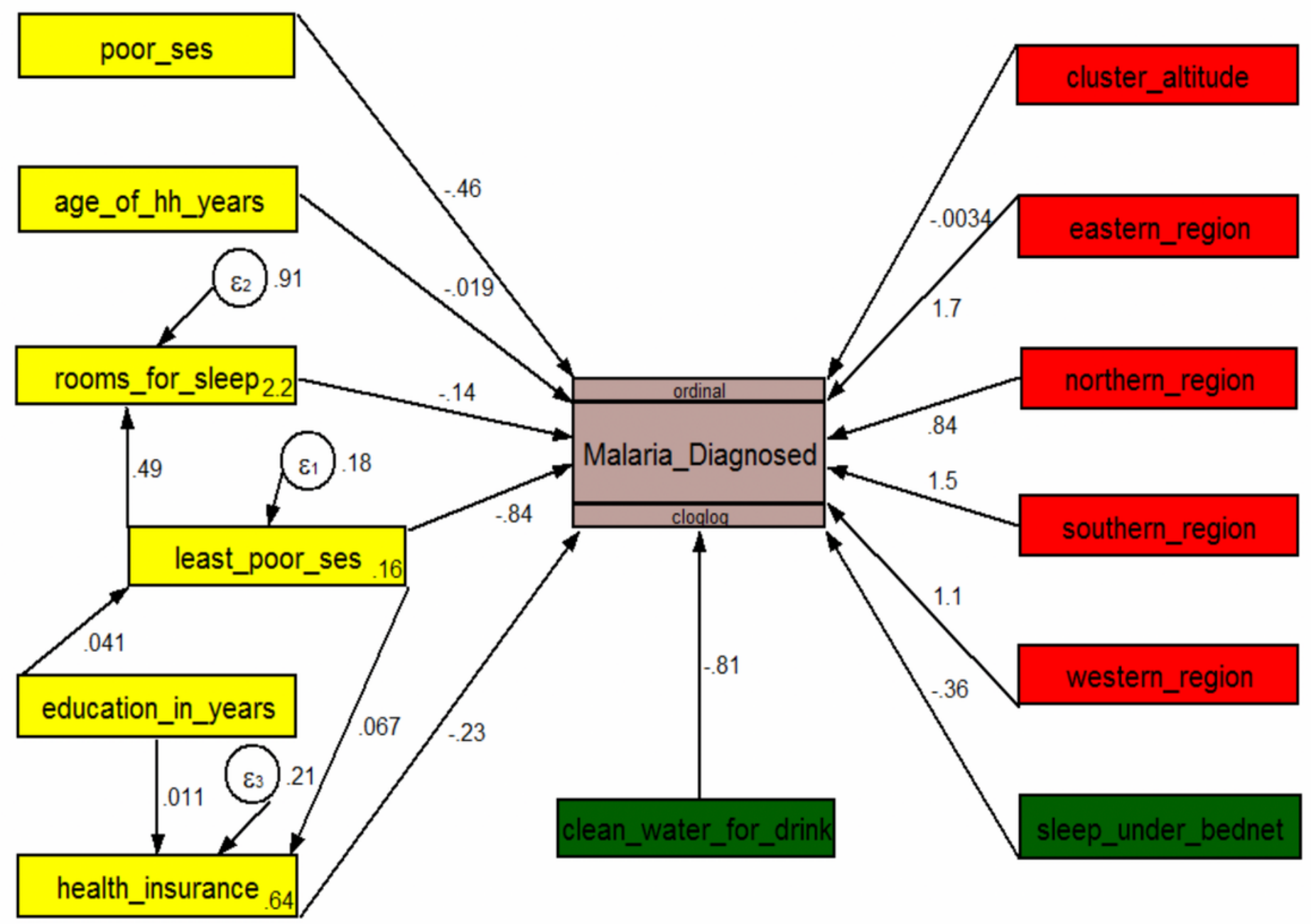

Figure 5

Diagram shows direct and indirect pathways and coefficients from G-SEM based ordinal regression analysis of the effects of selected random predictor variables on malaria infection outcome variable result in children and adults in Rwanda in 2010. Key for variables names: individuals covered by health insurance (health_insurance), number of rooms for sleeping in each house (rooms_for_sleeping), age of household members in years (age_of_hh_years), poor SES versus most poor SES (poor_ses), least poor SES versus most poor SES (least_poor_ses), education gain of each household member in years (education_in_years), number of household members sleep under treated mosquito bed net (sleep_under_bednet), household members have a clean water excess for drinking (had_water_excess), cluster altitude in meters (cluster altitude), eastern region of Rwanda (eastern_region), northern region of Rwanda (northern_region), western region of Rwanda (western_region), southern region of Rwanda (southern_region). Other: the arrows pointing from the exogenous (explanatory variables) to endogenous (dependent variable) and the error terms $(€)$ placed on all three endogenous variables.

\section{Supplementary Files}

This is a list of supplementary files associated with this preprint. Click to download.

- SupportingMaterialS5StataCommadns.docx

- SupporingMaterialAppendixS1S2S3S4.docx 